\title{
Matriciamento em saúde mental na Atenção Primária: uma revisão crítica (2000-2010)
}

\author{
Mental health matrix support in Primary Care: a critical review (2000-2010) \\ Apoyo matricial de la salud mental en Atención Primaria: una revisión crítica (2000-2010)
}

Karen Athié. Universidade do Estado do Rio de Janeiro (UERJ). karensathie@gmail.com (Autora corrrespondente)

Sandra Fortes. Universidade do Estado do Rio de Janeiro (UERJ). sandrafortes@gmail.com

Pedro Gabriel Godinho Delgado. Universidade Federal do Rio de Janeiro (UFRJ). pedrogabrieldelgado@gmail.com

\section{Resumo}

Objetivo: Analisar artigos publicados entre 2000 e 2010 que versam sobre a articulação entre saúde mental e Atenção Primária no Brasil, na perspectiva da prática do matriciamento. Método: Revisão em banco de dados virtual, no período referido. Os descritores foram: "saúde mental", "atenção primária", "atenção básica", "apoio matricial" e "matriciamento" e as categorias de análise foram construídas a partir da leitura dos artigos. Resultados: Foram selecionados 86 artigos, divididos em 3 categorias: a articulação analisada a partir da saúde mental, a partir da Atenção Primária e experiências de matriciamento. A análise evidenciou a existência de diferentes formas de descrever o trabalho em colaboração. Conclusões: A experiência brasileira está em consonância com as recomendações mundiais rumo à diminuição da lacuna terapêutica entre a oferta e as necessidades de cuidado. A construção da Rede de Saúde Mental integrada à Estratégia Saúde da Família é um novo desafio para a reforma psiquiátrica brasileira.

\section{Abstract}

Objective: A critical review of articles published between 2000 and 2010 on the relation between mental health and primary care in Brazil in view of the practice of matrix support. Method: Review of virtual database in the period in question. The descriptors were "mental health", "primary care", "matrix support", and the analysis categories were constructed from the reading of the articles. Results: We selected 86 articles, divided in three categories: the articulation analyzed from the Mental Health, the Primary Care Health, and experiences of matrix support. This analysis revealed the existence of different ways of describing the collaborative care. Conclusions: The Brazilian experience is in line with global recommendations towards decreasing the gap between the offer and treatment needs care. The construction of the integrated mental health network for Family Health Strategy is a new challenge for the Brazilian Psychiatric Reform.

\section{Resumen}

Objetivo: Analizar los artículos publicados entre 2000 y 2010 sobre la relación entre salud mental y atención primaria en el Brasil, desde el punto de vista del apoyo matricial. Método: Revisión de base de datos virtual, en el período en cuestión. Los descriptores fueron: "salud mental", "atención primaria", "atención básica!, "apoyo "matricial". Se determinaron categorías de análisis a partir de la lectura de los artículos. Resultados: Se seleccionaron 86 artículos, divididos en tres categorías: la articulación analizada desde la Salud Mental, desde la Atención Primaria y desde experiencias de apoyo matricial. El análisis reveló la existencia de diferentes maneras de describir el trabajo colaborativo. Conclusiones: la experiencia de Brasil está en concordancia con las recomendaciones internacionales sobre la reducción de la brecha entre la oferta y la atención de las necesidades terapéuticas. La construcción de la Red de Salud Mental integrada a la Estrategia de Salud Failiar es un nuevo reto para la Reforma Psiquiátrica brasileña.

\section{Palavras-chave:}

Serviços de Saúde Mental Atenção Primária Integração Trabalho Compartilhado Matriciamento

Keywords: Mental Health Services Primary Care Integrative Care Collaborative Care Matrix Support

\section{Palabras clave:} Servicios de Salud Mental Atención Primaria Integración Trabajo Compartido Apoyo Matricial
Fonte de financiamento: FAPERJ. 


\section{Introdução}

A alta prevalência dos transtornos mentais em contraponto com a escassez de serviços é um problema de saúde pública. As avaliaçôes mundiais apontam a necessidade de desenvolvimento dos sistemas de saúde, já que a taxa dos que necessitam e não são tratados é elevada ${ }^{1}$, sobretudo nos países em desenvolvimento ${ }^{2}$. A taxa média de falta de tratamento para a esquizofrenia é de 32,2\%, de 56,3\% para a depressão, de 56,0\% para a distimia, de 50,2\% para os transtornos bipolares, de 55,9\% para o transtorno de pânico, de 57,5\% para a ansiedade generalizada, de 57,3\% para o transtorno obsessivo-compulsivo e de $78,1 \%$ para abuso e dependência de álcool ${ }^{2}$. Considera-se que para haver a melhora desejada em relação à iniquidade e à oferta de serviços é preciso identificar o que afeta a implementaçấo dos recursos de: política e infraestrutura, serviços de saúde mental, serviços comunitários, recursos humanos e financeiros ${ }^{2}$. O estigma social ${ }^{3}$ da doença mental é considerado um dos fatores que afetam o desenvolvimento do plano de cuidado.

A Organização Mundial de Saúde (OMS) e pesquisadores do Movement for Global Mental Health (MGMH) argumentam em favor de um sistema de serviços pela base comunitária, conforme discussão publicada em edição especial no The Lancet Series ${ }^{4}$, em 2007. Nessa, é defendida uma rede efetiva de cuidados com um conjunto diversificado de serviços que inclua a Atenção Primária à Saúde (APS). A base racional é econômica e de efetividade e compreende que apenas os serviços especializados em saúde mental não garantirão a integralidade da assistência 5 .

O MGMH avaliou o impacto das doenças mentais na qualidade de vida dos indivíduos e na economia mundial. No início deste século, 450 milhóes de pessoas sofriam de desordens neuropsiquiátricas no mundo. Quando esse número é comparado com os dados dos anos 1990, a expectativa é que em 2020 haja um aumento de $15 \%$. Além disso, os transtornos mentais são responsáveis por $12 \%$ a $15 \%$ da incapacidade mundial total, mais do que as doenças cardiovasculares e duas vezes mais que o câncer ${ }^{5}$.

Esses problemas inspiraram a criação do slogan proposto pela OMS: "Não há saúde sem saúde mental” A discussão sobre a integralidade e os modelos médicos de atenção à saúde pode ser considerada um dos vetores para a diminuição da lacuna terapêutica entre a oferta de serviços de saúde mental e a necessidade da população ${ }^{8}$.

Um documento que retrata como pode ser complexo estruturar sistemas de saúde para os cuidados básicos da saúde global e mental é Integração da saúde mental nos cuidados de saúde primários: uma perspectiva global. A publicação realizada pela Organização Mundial de Saúde (OMS) e a Organização Mundial dos Médicos de Família (World Organization of Family Doctors/WONCA), em 2008, descreve 12 experiências diferentes, apontando as necessidades gerais e especificidades para essa integração. Os relatos esclarecem que não há uma abordagem única que possa ser seguida por todos os países ${ }^{9}$ em razão das diferenças locais e culturais, o que pode ser verificado nos princípios gerais sugeridos para tal integração (Tabela 1).

Tabela 1. 10 Princípios para integrar a saúde mental nos cuidados primários.

\begin{tabular}{ll}
$\mathbf{1}$ & A política e os planos têm que incorporar cuidados primários para a saúde mental. \\
$\mathbf{2}$ & Ativismo e campanhas de informação são necessários para mudar atitudes e comportamentos. \\
$\mathbf{3}$ & É necessária a formação adequada dos profissionais de cuidados primários. \\
$\mathbf{4}$ & As tarefas de cuidado primário têm que ser limitadas e realizáveis. \\
$\mathbf{5}$ & Profissionais e instituições especializadas em saúde mental devem estar disponíveis para apoiar os cuidados primários. \\
$\mathbf{6}$ & Os pacientes devem ter acesso a medicamentos psicotrópicos essenciais nos cuidados primários. \\
$\mathbf{7}$ & A integração é um processo, não um acontecimento. \\
$\mathbf{8}$ & Um coordenador de serviços de saúde mental é crucial. \\
$\mathbf{9}$ & A colaboração com outros setores governamentais não relacionados com a saúde, com organizações não governamentais, com profissionais de saúde \\
$\mathbf{1 0}$ & ao nível da vila ou da comunidade e com voluntários é necessária. \\
\hline
\end{tabular}

Fonte: WHO e WONCA9 . Integração da saúde mental nos cuidados de saúde primários: Uma perspectiva global, 2009. 


\section{As recomendações mundiais e as especificidades locais}

A diversidade dos cenários onde ocorre a articulação entre a Saúde Mental e a APS permite a observação da interferência cultural, geográfica e política local. Assim, ainda que a direção de cuidado seja a mesma, verificam-se diferenças nas práticas, a começar pela forma de nomeá-las: 1) práticas integrativas ou de integração (integrative care) ${ }^{10}, 2$ ) trabalho em colaboração (collaborative care) ${ }^{11}$, 3) trabalho compartilhado (shared care) $\left.{ }^{12}, 4\right)$ matriciamento $^{13}$ e apoio matricial ${ }^{14}$.

A realidade local é determinante para o modelo de articulação. Duas importantes experiências, consideradas referências mundiais, inclusive para o sistema brasileiro, são: a dos canadenses pelos Family Doctors (FD) e a dos britânicos através dos General Practitioners (GP). Um exemplo é a realização da 12a Conferência de Cuidados Colaborativos em Saúde Mental pelos médicos de família no Canadá, cujo tema é Charting a New Course to Better Care: stronger Links between Consumers, Families and Health Care Providers ${ }^{15}$.

\section{Definição da articulação entre a saúde mental e a APS: matriciamento}

No Brasil a definição de matriciamento em saúde mental tem origem em discussóes sobre a organização do trabalho em saúde nos anos $1990^{16}$, quando a construção da sua rede de serviços se inicia, depois da aprovação do SUS em 1988. Naquele momento, as referências que se opunham ao funcionamento da saúde centrado no hospital eram as Unidades Básicas de Saúde (UBS). Com o desenvolvimento das políticas e do SUS, a rede de saúde começa a incluir em seu sistema, ainda nos anos 1990, equipamentos como os Centros de Atenção Psicossocial (CAPS) e o Programa Saúde da Família (PSF), em 1994. O primeiro citado, representando o tratamento não hospitalocêntrico para pacientes psiquiátricos e o segundo inspirado na discussão da Reforma Sanitária Brasileira para a saúde geral da população. Nos primeiros anos do século XXI, as políticas públicas desses equipamentos foram aprovadas sob a forma da Lei n. 10.216, de 2001 (CAPS), e da Portaria n. 648, de 2006 (PSF). A noção de matriciamento em saúde mental e da articulação entre serviços, necessária para o funcionamento da rede é formalizada através da criação dos Núcleos de Apoio à Saúde da Família (NASF), pela Portaria n. 154/2008 ${ }^{17}$, que recomenda a presença de profissional de saúde mental para o trabalho em colaboração, redimensionando o trabalho de referência e contrarreferência e incluindo nas açóes a necessidade de pensar essa nova forma de articulação 20 anos após a criaçáo do SUS e quase 10 anos após a promulgação da Lei n. 10.216/2001 ${ }^{18}$. Além disso, o NASF formaliza o cálculo dos gastos em saúde mental na APS, o que causa um impacto no financiamento geral da saúde mental ${ }^{19}$.

Este artigo é resultado da revisão de literatura feita pela pesquisa Avaliação do cuidado em Saúde Mental na Atenção Primária na Área Programática 2.2 do Município do Rio de Janeiro, aprovada em 2010 pela FAPERJ e que tem como objetivo analisar criticamente artigos publicados entre 2000 e 2010 que versam sobre a articulação entre Saúde Mental e APS no Brasil.

\section{Método}

A revisão crítica ${ }^{20}$ com delineamento descritivo foi desenvolvida através da literatura científica concentrando-se nos trabalhos publicados sobre a articulação entre a saúde mental e a APS entre 2000 a 2010 no Brasil. Essa escolha foi baseada nas recomendações da Base Cochrane de revisões sistemáticas qualitativas, onde esse tipo de análise explora aspectos políticos e práticos de propostas recentes para a saúde. Dois elementos que enfatizam esse tipo de estudo são: o levantamento de informaçóes sobre o problema e a sua organização e definição de efetividade ${ }^{21}$.

O mapeamento da literatura foi realizado prioritariamente através de consulta pela Virtual Health Library (www.bireme. br). A amplitude da busca também incluiu a Latin American and Caribbean Health Sciences (LILACS), Base COCHRANE, Scientifc Eletronic Library (SCIELO pública) e Medline de artigos em português ou sobre a experiência brasileira. A estratégia de busca foi a combinação dos termos: "saúde mental e atenção primária"; "saúde mental e atenção básica"; "saúde mental e matriciamento; e "saúde mental e apoio matricial". Da combinação dos descritores "saúde mental e atenção básica" foram encontrados 4.989 artigos, dos quais 205 em português. A segunda combinação, "saúde mental e atenção primária", resultou em 5.523 artigos, dos quais 187 em português. Esses modos de busca se justificam pelo uso de terminologias utilizadas no Brasil, onde Atenção Básica é a denominação mais comum. A utilização das combinaçóes "saúde mental" e as 
palavras "matriciamento" e "apoio matricial" foi em razão da terminologia técnica que vem sendo utilizada no Brasil. Para a combinação "saúde mental" com a palavra "matriciamento" foram encontradas 4 referências e com a expressão "apoio matricial", 7, todas em português. Assim, dos 10.523 documentos catalogados, essa revisão selecionou as 403 referências escritas em língua portuguesa.

Os critérios de avaliação das 403 referências registradas e elegíveis para as finalidades deste estudo foram:

- Inclusão: para os artigos de revista que citavam a integração entre os cuidados realizados na Atenção Básica e a saúde mental no período de janeiro de 2000 a dezembro de 2010;

- Exclusão: a) para as repetiçôes dos textos incluídos nos modos de busca utilizados; b) monografias, teses, dissertaçóes; também foram eliminados documentos encontrados na busca que não tinham relevância na discussão entre a integração saúde mental e Atenção Primária, ou documentos técnicos.

\section{Resultados}

Entre janeiro de 2000 e dezembro de 2010 foram selecionados 86 artigos (Tabela 2) sobre a articulação entre a Saúde Mental e a Atenção Básica. Foi observado que a maioria desses artigos foi produzida nas regióes Sudeste, Sul e em algumas cidades do Nordeste. No período estudado, observou-se tendência ao aumento da produçáo científica sobre o assunto a partir dos anos de 2006 e 2007, culminando com o incremento significativo da produção nos anos de 2008 e 2009 , como se pode verificar na Figura 1.

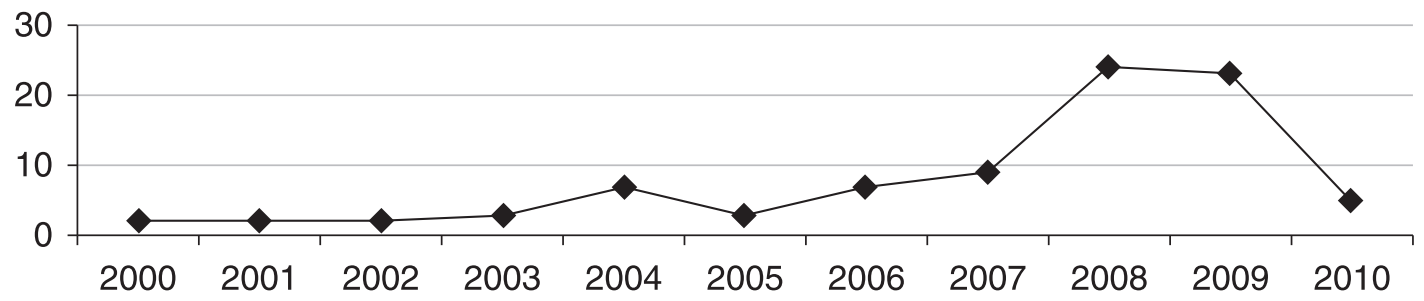

Figura 1. Incremento anual da Produção de artigos publicados sobre saúde mental e atenção primária entre 2000 e 2010.

As categorias de análise foram criadas através da leitura de características comuns. Os itens: categoria profissional e campo assistencial foram determinantes, pois foi observado que os autores discutiam de dois modos: a partir da experiência prática e/ou a partir do seu referencial específico, tanto do campo da saúde mental como do da Atenção Primária. Assim foram criadas três categorias: articulação vista da saúde mental, articulação vista da Atençáo Primária e articulação realizada na prática, que é quando o matriciamento acontece.

\section{Articulação vista da saúde mental: a visão dos especialistas em saúde mental}

Os 28 artigos incluídos nessa categoria são de análises qualitativas. Os enfoques são: aspectos políticos, históricos, dos recursos estruturais e humanos da rede e de avaliação. Os principais assuntos identificados foram: avaliação de modelo de atenção especializada na Rede Básica em Saúde Mental, formação dos trabalhadores de saúde mental, aspectos operacionais da desinstitucionalização hospitalar de pessoas com transtornos mentais graves e persistentes e o trabalho do CAPS, atenção especializada em saúde mental no território, centros de convivência, articulação teórica dos princípios da Saúde Mental e da Atenção Básica, Políticas de Saúde Infantil, depressão e uso de drogas, relato de experiências dos profissionais de saúde mental, avaliações de serviço, estudos no campo das representaçóes e políticas educacionais. Essa leitura observa que as publicações desse período de 10 anos sobre a articulação entre a Saúde Mental e a Atenção Primária pelo olhar dos especialistas de saúde mental se relaciona ao processo de reforma psiquiátrica e a ampliação do modelo assistencial não hospitalocêntrico. A suposição é que a articulação entre a Saúde Mental e a APS é pensada pela referência prática nas Unidades Básicas de Saúde, Ambulatórios Especializados e CAPS muito mais do que pela Estratégia da Saúde da Família. Nessa perspectiva, observa-se que os CAPS e os ambulatórios de saúde mental localizados nas UBS são considerados APS, embora sejam espaços de atenção por especialistas. 
Tabela 2. Artigos selecionados sobre a articulação entre a Saúde Mental e a Atenção Básica.

\section{Lista dos 86 artigos revisados}

\section{Articulação vista da saúde mental: a visão dos especialistas em saúde menta}

Boarini ML, Borges RF. 0 Psicólogo na atenção básica à saúde. Psicol Cienc Prof. 2009; 29(3): 602-613. http://dx.doi.org/10.1590/S1414-98932009000300013

Campos TRO, Furtado JP, Passos E, Ferrer AL, Miranda L, Gama CAP. Avaliação da rede de centros de atenção psicossocial: entre a saúde coletiva e a saúde mental. Rev Saúde Pública. 2009; 43(Suppl. 1): 16-22. http://dx.doi.org/10.1590/S0034-89102009000800004

Caçapava JR, Colvero LA, Martines WRV, Machado AL, Silva ALA, Vargas D, et al. Trabalho na atenção básica: integralidade do cuidado em saúde mental. Rev Esc Enferm USP. 2009; 43(Spe. 2): 1256-1260. http://dx.doi.org/10.1590/S0080-62342009000600019

Gama CAP, Onocko Campos R. Saúde Mental na Atenção Básica: Uma pesquisa bibliográfica exploratória em periódicos de saúde coletiva (1997-2007). Cad Bras Saúde Mental. 2009 out./dez; 1(2): 112-131.

Dalla Vecchia M, Martins STF. Desinstitucionalização dos cuidados a pessoas com transtornos mentais na atenção básica: aportes para a implementação de ações. Interface. 2009; 13(28): 151-164. http://dx.doi.org/10.1590/\$1414-32832009000100013

Escudeiro CC, Souza ML. A Saúde mental no Sistema Único de Saúde: mudança do modelo de atenção na região de Lins-SP. Saúde Soc. 2009; 18(Suppl. 1): 44-47. http://dx.doi.org/10.1590/S0104-12902009000500007

Ferreira Neto J. Psicologia e saúde mental: três momentos de uma história. Saúde Debate. 2008 jan./dez.; 32(78-80): 18-26.

Hirdes A. A reforma psiquiátrica no Brasil: uma (re)visão. Ciênc Saúde Coletiva. 2009; 14(1): 297-305. http://dx.doi.org/10.1590/\$1413-81232009000100036 Nascimento A, Galvanese AT. Avaliação da estrutura dos centros de atenção psicossocial do município de São Paulo, SP. Rev Saúde Pública. 2009; 43(Supl. 1):8-15 http://dx.doi.org/10.1590/S0034-89102009000800003

Silveira DP, Vieira ALS. Saúde mental e atenção básica em saúde: análise de uma experiência no nível local. Ciênc Saúde Coletiva. 2009; 14(1): 139-148. http://dx.doi. org/10.1590/S1413-81232009000100019

Borges PL, Bretas RP, Azevedo SF, Barbosa JMM. Perfil dos idosos freqüentadores de grupos de convivência em Belo Horizonte, Minas Gerais, Brasil. Cad Saúde Pública. 2008; 24(12): 2798-2808. http://dx.doi.org/10.1590/S0102-311X2008001200008

Botti NC, Andrade WV. A saúde mental na atenção básica - articulação entre os princípios do SUS e da reforma psiquiátrica. Rev Bras Enferm. 2009 set./out; 62(5):

$675-80$.

Couto MCV, Duarte C, Delgado PGG. A saúde mental infantil na Saúde Pública brasileira: situação atual e desafios. Rev Bras Psiquiatr. 2008; 30(4): 384-389. http:// dx.doi.org/10.1590/S1516-44462008000400015

Nunes M, Torrenté M, Ottoni V, Moraes Neto V, Santana M. A dinâmica do cuidado em saúde mental: signos, significados e práticas de profissionais em um Centro de Assistência Psicossocial em Salvador, Bahia, Brasil. Cad Saúde Pública. 2008; 24(1): 188-196. http://dx.doi.org/10.1590/S0102-311X2008000100019 Reinaldo AMS. Saúde mental na atenção básica como processo histórico de evolução da psiquiatria comunitária. Esc Anna Nery. 2008 mar.; 12(1): 173-178. http:// dx.doi.org/10.1590/S1414-81452008000100027

Scheneider A. A construção da rede de atenção em saúde mental de um município do Sul do Brasil. Barbarói. 2008 jan./jun.; 28: 124-135.

Tonini NS, Maraschin MS, Kantorski LP. Reorganização dos serviços: desafios para efetivação da Reforma Psiquiátrica. Rev Gaúcha Enferm. 2008 jun.; 29 (2):262-8. Vasconcelos MGF, Jorge MSB, Guimarães JMX, Pinto A. Saúde mental no contexto do programa saúde da família: representações sociais de usuários e familiares. Rev. RENE. 2008 jul./set.; 9(3): 9-18.

Zambenedetti G, Perrone C. 0 Processo de construção de uma rede de atenção em Saúde Mental: desafios e potencialidades no processo de Reforma Psiquiátrica. Physis. 2008; 18(2): 277-293. http://dx.doi.org/10.1590/S0103-73312008000200005

Braga SG, Morais M. Queixa escolar: atuação do psicólogo e interfaces com a educação. Psicol USP. 2007; 18(4): 35-51. http://dx.doi.org/10.1590/S0103-

65642007000400003

Fontanella B, Turato ER. Percepção de sintomas depressivos por dependentes de substâncias psicoativas procurando tratamento. J Bras Psiquiatr. 2005 out./dez.; $54(4)$ : 278-284

Leite L, Oliveira M. 0 trabalho da equipe itinerante nos processos de inclusão social de portadores de sofrimento psíquico. Divulg Saúde Debate. 2006 ago.; 36: 53-60. Costa-Rosa A, Luzio C, Mendes M, Florezi P. Uma experiência de pronto atendimento em saúde mental coletiva. Estud Psicol. 2004; 21(2): 101-115. http://dx.doi. org/10.1590/S0103-166X2004000200008

De Micheli D, Fisberg M, Formigoni MLOS. Estudo da efetividade da intervenção breve para o uso de álcool e outras drogas em adolescentes atendidos num serviço de assistência primária à saúde. Rev Assoc Med Bras. 2004; 50(3): 305-313. http://dx.doi.org/10.1590/S0104-42302004000300040

Sousa H. A saúde mental em Paraipaba (CE). Divulg Saúde Debate. 2004 mar.; (30): 91-94.

Campos C, Soares CB. The production of mental health services: the conception of the workers. Ciênc Saúde Coletiva. 2003; 8(2): 621-628. http://dx.doi.org/10.1590/ S1413-81232003000200022

Delgado PG, Weber R. Configurações dos serviços de atenção diária até 2001: um estudo com 295 serviços. J Bras Psiquiatr. 2003 maio-jun.; $52(3): 236-242$.

Breda M, Augusto L. 0 cuidado ao portador de transtorno psíquico na atenção básica de saúde. Ciênc Saúde Coletiva. 2001; 6(2): 471-480. http://dx.doi.org/10.1590/

S1413-81232001000200016

\section{Articulação vista da Atenção Primária: a visão dos não especialistas em saúde mental}

Andrade FB, Ferreira Filha MO, Dias MD, Silva AO, Costa ICC, Lima ÉAR, et al. Promoção da saúde mental do idoso na atenção básica: as contribuições da terapia comunitária. Texto Contexto Enferm. 2010 mar.; 19(1): 129-136. 
Tabela 2. Continuação...

\section{Lista dos 86 artigos revisados}

Braga LC, Carvalho LR, Binder M. Condições de trabalho e transtornos mentais comuns em trabalhadores da rede básica de saúde de Botucatu (SP). Ciênc Saúde Coletiva. 2010; 15 (Suppl. 1): 1585-1596. http://dx.doi.org/10.1590/S1413-8123201000070007

Neves H, Lucchese R, Munari D. Saúde mental na atenção primária: necessária constituição de competências. Rev Bras Enferm. 2010; 63(4): 1585-1596. http://dx.doi. org/10.1590/S1413-81232010000700070

Ribeiro L, Medeiros SM, Albuquerque JS, Fernandes SMBA. Saúde mental e enfermagem na estratégia saúde da família: como estão atuando os enfermeiros?. Rev Esc Enferm USP. 2010; 44(2): 376-382. http://dx.doi.org/10.1590/S0080-62342010000200019

Rosenstock K, Neves M. Papel do enfermeiro da atenção básica de saúde na abordagem ao dependente de drogas em João Pessoa, PB, Brasil. Rev Bras Enferm. 2010 ago.; 63(4): 581-586. http://dx.doi.org/10.1590/S0034-71672010000400013

Andrade F, Bezerra AIC, Pontes ALF, Ferreira Filha MO, Vianna RPT, Dias MD, et al. Saúde mental na atenção básica: um estudo epidemiológico baseado no enfoque de risco. Rev Bras Enferm. 2009; 62(5): 675-680. http://dx.doi.org/10.1590/S0034-71672009000500004

Tanaka 0, Ribeiro E. Ações de saúde mental na atenção básica: caminho para ampliação da integralidade da atenção. Ciênc Saúde Coletiva. 2009; 14(2): 477-486. http://dx.doi.org/10.1590/S1413-81232009000200016

Fiorotti C, Tomazelli J, Malagris L. Transtornos mentais comuns em pacientes hipertensos:estudo em unidade de atenção primária à saúde no Rio de Janeiro. Rev APS. 2009 jul./set.; 12(3).

Mascia A, Silva FB, Lucchese AC, Marco MA, Martins MCFN, Martins LAN. Atitudes frente a aspectos relevantes da prática médica: estudo transversal randomizado com alunos de segundo e sexto anos. Rev Bras Educ Med. 2009; 33(1): 40-48. http://dx.doi.org/10.1590/S0100-55022009000100006

Rodrigues MAP, Facchini LA, Piccini RX, Tomasi E, Thumé E, Silveira DS, et al. Use of primary care services by elderly people with chronic conditions, Brazil. Rev Saúde Pública. 2009; 43(4): 604-612. http://dx.doi.org/10.1590/S0034-89102009005000037

Romagnoli R. Breve estudo institucionalista acerca do Programa de Saúde da Família. Saúde Soc. 2009; 18(3): 525-536. http://dx.doi.org/10.1590/S0104-

12902009000300016

Vecchia MD, Martins STF. Concepções dos cuidados em saúde mental por uma equipe de saúde da família, em perspectiva histórico-cultural. Ciênc Saúde Coletiva. 2009; 14(1): 183-193. http://dx.doi.org/10.1590/S1413-81232009000100024

Eglê K. Atendimento ao comportamento suicida: concepções de enfermeiras de unidades de saúde. Ciênc Cuid Saúde. 2008 out./dez.; 7(4): 468-475.

Fernandes R, Rozenthal M. Avaliação da sintomatologia depressiva de mulheres no climatério com a escala de rastreamento populacional para depressão CES-D. Rev Psiquiatr Rio Gd Sul. 2008; 30(3): 192-200. http://dx.doi.org/10.1590/S0101-81082008000400008

Fonseca M, Guimarães M, Vasconcelos E. Sofrimento difuso e transtornos mentais comuns: uma revisão bibliográfica. Rev APS. 2008 jul./set.; 11(3): $285-294$.

Gianini R, Carvalho TC, Dos Anjos RMP, Pinto PLS, Maluf ME, Lanza LB, et al. Prática de rastreamento no cenário do Programa Saúde da Família em Sorocaba (SP). Rev Bras Educ Med. 2008; 32(1): 15-22. http://dx.doi.org/10.1590/S0100-55022008000100003

Gonçalves DM, Kapczinski F. Prevalência de transtornos mentais em indivíduos de uma unidade de referência para Programa Saúde da Família em Santa Cruz do Sul, Rio Grande do Sul, Brasil. Cad Saúde Pública. 2008; 24(9): 2043-2053. http://dx.doi.org/10.1590/S0102-311X2008000900010

Paniz VMV, Fassa AG, Facchini LA, Bertoldi AD, Piccini RX, Tomasi E, et al. Acesso a medicamentos de uso contínuo em adultos e idosos nas regiões Sul e Nordeste do Brasil. Cad Saúde Pública. 2008; 24(2): 267-280. http://dx.doi.org/10.1590/S0102-311X2008000200005

Ribeiro MS, Alves MJM, Silva PM, Vieira E. Concordância diagnóstica entre profissionais dos níveis primário e secundário na atenção à saúde mental. Rev APS. 2008 abr./jun.; 11(2): 126-135.

Rodrigues MP, Lima KC, Roncalli A. A representação social do cuidado no programa saúde da família na cidade de Natal. Ciênc Saúde Coletiva. $2008 ; 13(1): 71-82$. http://dx.doi.org/10.1590/S1413-81232008000100012

Vargas D, Luis M. Alcohol, alcoholism and alcohol addicts: conceptions and attitudes of nurses from district basic health centers. Rev Latino-Am Enferm. 2008; 16: 543-550. http://dx.doi.org/10.1590/S0104-11692008000700007

Ferriolli SHT, Marturano E, Puntel L. Family context and child mental health problems in the Family Health Program. Rev Saúde Pública. 2007; 41(2): 251-259. http:// dx.doi.org/10.1590/S0034-89102006005000017

Fortes S, Villano L, Lopes CS. Nosological profile and prevalence of common mental disorders of patients seen at the Family Health Program (FHP) units in Petrópolis, Rio de Janeiro. Rev Bras Psiquiatr. 2008 mar.; 30(1): 32-37. http://dx.doi.org/10.1590/S1516-44462006005000066

Lemos S, Lemos M, Souza MGG. 0 preparo do enfermeiro da atenção básica para a saúde mental. Arq Ciênc Saúde 2007 out./dez; 14(4): $198-202$.

ModestoT, Santos D. Saúde mental na atenção básica. Rev Bahiana Saúde Pública. 2007 jan./jun.; 31(1): 19-24.

Barros M, Pillon S. Programa saúde da família: desafios e potencialidades frente ao uso de drogas. Rev Eletr Enferm. 2006; 8(1): 144-149

Leal S, Lopes M. Vulnerabilidade à morbidade por causas externas entre mulheres com 60 anos e mais, usuárias da atenção básica de saúde. Ciênc Cuid Saúde. 2006 set./dez.; 5(3): 309-316.

Tanaka 0, Lauridsen-Ribeiro E. Desafio para a atenção básica: incorporação da assistência em saúde mental. Cad Saúde Pública. 2006; 22(9): 1845-1853. http://dx.doi. org/10.1590/S0102-311X2006000900015

Tavares C. A educação permanente da equipe de enfermagem para o cuidado nos serviços de saúde de saúde mental. Texto Contexto Enferm. 2006 abr./jun.; 15(2):

287-295. http://dx.doi.org/10.1590/S0104-07072006000200013 
Tabela 2. Continuação...

\section{Lista dos $\mathbf{8 6}$ artigos revisados}

Oliveira A, Ataide I, Silva M. A invisiblidade dos problemas de saúde mental na atenção primária: o trabalho da enfermeira construindo caminhos. Texto Contexto Enferm. 2004; 13(4): 618-624. http://dx.doi.org/10.1590/S0104-07072004000400015

Valentini W, Levav I, Kohn R, Miranda CT, Mello A AF, Mello MF, et al. Treinamento de clínicos para o diagnóstico e tratamento da depressão. Rev Saúde Pública. 2004; 38(4): 523-528. http://dx.doi.org/10.1590/S0034-89102004000400007

Dytz J, Lima M, Rocha S. 0 modo de vida e a saúde mental de mulheres de baixa renda. Saúde Debate. 2003 jan./abr.; 27(63): 15-24.

Enumo S, Trindade Z. Ações de prevenção da deficiência mental, dirigidas a gestantes e recém-nascidos, no âmbito da saúde pública da grande Vitória-ES. Psicol USP; 2002; 13(1): 107-132. http://dx.doi.org/10.1590/S0103-65642002000100006

Fleck M, Lima AFBS, Louzada S, Schestasky G, Henriques A, Borges VR, et al. Associação entre sintomas depressivos e funcionamento social em cuidados primários à saúde. Rev Saúde Pública. 2002; 36(4): 431-438. http://dx.doi.org/10.1590/S0034-89102002000400008

Lima RT, Mangueira NM. Avaliação de implantação de ações básicas de saúde. Rev Bras Ciênc Saúde. 2001; 5(2): 149-158.

Falceto 0, Busnello E, Bozzetti MC. Validação de escalas diagnósticas do funcionamento familiar para utilização em serviços de atenção primária à saúde. Rev Panam Salud Publica. 2000; 7(4): 255-263. http://dx.doi.org/10.1590/S1020-49892000000400007

Dutra K, Perini E, Starling S. Utilizaçäo do diazepam em unidades de atençäo primária do distrito sanitário norte - secretaria municipal de saúde de Belo Horizonte, MG,

1997. Rev Ciênc Farm. 2000; 21(2): 201-215.

\section{Articulação do ponto de vista do matriciamento}

Arona E. Implantação do matriciamento nos serviços de saúde de Capivari. Saúde Soc. 2009; 18(Suppl. 1): 26-36. http://dx.doi.org/10.1590/S0104-

12902009000500005

Carneiro A, Oliveira ACM, Santos MMS, Alves MS, Casais NA, Santos JE. Saúde mental e atenção primária: uma experiência com agentes comunitários de saúde em Salvador-BA. Rev Bras Promoç Saúde. 2009 out./dez.; 22(4): 264-271. http://dx.doi.org/10.5020/18061230.2009.p264

Delfini P, Sato MT, Antoneli P, Guimarães P. Parceria entre CAPS e PSF: o desafio da construção de um novo saber. Ciênc Saúde Coletiva. 2009; 14(Suppl. 1): 1483-

1492. http://dx.doi.org/10.1590/S1413-81232009000800021

Dimenstein M, Severo AK, Brito M, Pimenta AL, Medeiros V, Bezerra E. 0 apoio matricial em Unidades de Saúde da Família: experimentando inovações em saúde mental. Saúde Soc. 2009; 18(1): 63-74. http://dx.doi.org/10.1590/S0104-12902009000100007

Figueiredo M, Campos Onocko R. Saúde Mental na atenção básica à saúde de Campinas, SP: uma rede ou um emaranhado?. Ciênc Saúde Coletiva. 2009; 14(1):

129-138. http://dx.doi.org/10.1590/S1413-81232009000100018

Paiva F, Ronzani T. A inserção do psicólogo na atenção primária à saúde: possibilidades e desafios do trabalho em saúde coletiva. Rev APS. 2009 jan./mar.; $12(1): 88-92$. Com apoio das Equipes de Saúde Mental, ESF de Florianópolis tratam transtornos leves na Atenção Básica. Rev Bras Saúde Fam. 2008 jul./set.; 9(19): 36-41. Bezerra E, Dimenstein M. Os CAPS e o trabalho em rede: tecendo o apoio matricial na atenção básica. Psicol Cienc Prof. 2008; 28(3): 632-645. http://dx. doi. org/10.1590/S1414-98932008000300015

Clemente A, Matos DR, Grejanin DKM, Santos HE, Quevedo MP, Massa PA. Residência multiprofissional em saúde da família e a formação de psicólogos para a atuação na atenção básica. Saúde Soc. 2008; 17(1): 176-184. http://dx.doi.org/10.1590/S0104-12902008000100016 Figueiredo Dorsa M, Onocko R. Saúde Mental e Atenção Básica à Saúde: 0 apoio matricial na construção de uma rede multicêntrica. Saúde Debate. 2008; 32(78-80): 143-150.

Soares M. A inserção do enfermeiro psiquiátrico na equipe de apoio matricial em saúde mental. SMAD, Rev Eletr Saúde Mental Álcool Drog (Ed. port.). 2008; 4(2): 2-12. Barban E, Oliveira A. 0 modelo de assistência da equipe matricial de saúde mental no Programa Saúde da Família do município de São José do Rio Preto. Arq Ciênc Saúde; 2007 jan-mar;14(1):54-65.

Nunes M, Juca V, Valemtim C. Ações de saúde mental no Programa Saúde da Família: confluências e dissonâncias das práticas com os princípios das reformas psiquiátrica e sanitária. Cad Saúde Pública. 2007; 23(10): 2375-2384. http://dx.doi.org/10.1590/S0102-311X2007001000012 Oliveira I, Silva F, Yamamoto 0. A psicologia no Programa de Saúde da Família (PSF) em Natal: espaço a ser conquistado ou um limite da prática psicológica? Aletheia. 2007 jan./jun.; 25: 5-19.

Tofoli LF, Fortes S. Apoio Matricial de Saúde Mental na Atenção Primária no Município de Sobral no Ceará: relato de experiência. Sanare. 2007; 6(2): 34-42. Breda M, Rosa W, Pereira M, Scatena M. Duas estratégias e desafios comuns: a reabilitação psicossocial e a saúde da família. Rev Latino-Am Enferm. 2005; 13(3): 450-452. http://dx.doi.org/10.1590/S0104-11692005000300021

Buchele F, Laurindo D, Borges V, Coelho E. A interface da saúde mental na Atenção Básica. Cogitare Enferm. 2006 set./dez.; 11(3): 226-233.

Santana M, Morais M. Saúde mental em Francisco Morato: percepção dos trabalhadores do CAPs sobre a atenção praticada e suas dificuldades. Bol Inst Saúde. 2005; 37: 31-33.

Souza A. Ampliando o campo da atenção psicossocial: a articulação dos centros de atenção psicossocial com a saúde da família. Esc Anna Nery. 2006 dez.; 10(4): 703-710. http://dx.doi.org/10.1590/S1414-81452006000400012

Ballester D. Avaliação de um programa de capacitação em saúde mental para médicos dos serviços básicos de saúde. São Paulo; 2004. Nascimento A, Braga V. Atenção em Saúde Mental: a prática do enfermeiro e do médico do Programa Saúde da Família de Caucaia - CE. Cogitare Enferm. 2004 jan./ jun.; $9(1): 84-93$. 


\section{Articulação vista da Atenção Primária: a visão dos não especialistas em saúde mental}

Os 37 artigos incluídos nessa categoria versam sobre: compreensão das fases da vida (pré-natal, infância, adolescência e velhice), questôes de gênero, contexto familiar, estudos de prevalência de transtornos mentais comuns e em comorbidade com outras doenças crônicas, avaliação de risco para transtornos mentais, adesão ao tratamento de doenças crônicas, queixas inespecíficas, qualidade de vida e estratégias terapêuticas comunitárias, drogas, álcool, suicídio, somatizaçôes, acesso à medicação, uso abusivo de benzodiazepínicos, saúde mental do trabalhador, capacitação para o diagnóstico em saúde mental, fluxo de atendimento, busca ativa e sistema de informaçóes. A perspectiva que une essas referências é a ideia de resolutividade em saúde mental pela equipe da Atenção Primária, onde os artigos traduzem as necessidades observadas na assistência e buscam soluçôes efetivas para os problemas apontados pela ação junto à população adscrita.

\section{Experiências de matriciamento: a visão a partir da relação entre especialistas e não especialistas em saúde mental}

Os 21 artigos incluídos nessa categoria relatam a experiência do trabalho em colaboração na assistência e na formação de recursos humanos. Trata-se do matriciamento em saúde mental como prática. As descriçôes apontam o cenário de relação entre o matriciador (especialista de saúde mental) e os matriciados (não especialistas em saúde mental) ${ }^{22,23}$.

Essa perspectiva da articulação, que se funda na experiência, difere em suas formas de organizar o trabalho pois, além da assistência, inclui a gestão, os pactos políticos e locais, assim como a avaliação política. Nesse espectro, a articulação é descrita a partir de experiências focais, onde as experiências descritas nascem de iniciativas pessoais dos profissionais da ponta e que, a partir da demanda da populaçáo, tratam das necessidades clínicas e circunstanciais. Também aparece entre as descrições a necessidade de atividades de educação permanente.

\section{Discussão}

Este levantamento bibliográfico ajudou a mapear diferenças de perspectiva política da Saúde Mental e da Atenção Primária que se relacionam ao mesmo tempo com vetores clínicos e históricos.

As evidências clínicas revelam necessidade de ampliação da cobertura em saúde mental em razão da alta prevalência de sofrimento psíquico identificada na Atenção Primária. Em 1995, a OMS ${ }^{24,25}$ relatou as formas mais frequentes: depressão (31\%), ansiedade (19\%), alcoolismo (10\%) e transtornos somatoformes (18\%). Dados semelhantes se confirmaram no Brasil. Em estudos ${ }^{26,27}$ realizados na década de 1980 foram encontradas prevalências aproximadas de $55 \%$ de transtornos mentais comuns nos pacientes de centros de saúde de Sáo Paulo ${ }^{28-30}$. Em estudo de $2004^{31}$, na clientela atendida no Programa Saúde da Família (PSF) em Petrópolis, RJ, foi verificada prevalência de 56\% de transtornos mentais comuns, entre os quais: depressão, ansiedade, transtorno de estresse pós-traumático, transtorno de dor somatoforme e transtornos dissociativos. Esses dados se referem à detecção por não especialistas e reforçam a necessidade de inclusão sistemática desse nível de cuidado no planejamento da assistência em saúde mental, como preconiza a OMS e o MGMH.

O outro vetor, e que se refere a questão histórica, pode relativizar as noçôes de Atenção Primária e até mesmo a de território. Isto se evidencia na medida em que os artigos incluídos na categoria "articulação vista da saúde mental" se circunscrevem ao campo dos serviços em saúde mental (CAPS e rede ambulatorial) contabilizados como especializados, além das ações na ESF. Em contrapartida, na categoria "articulação vista da Atenção Primária", a ênfase é essencialmente na atenção realizada pela ESF. Não existem estudos sobre o impacto dessa diferença de olhar da saúde mental e da Atenção Primária na organização dos sistemas de saúde; entretanto, estudo ${ }^{32}$ que compara Unidades de Saúde da Família (USF) - que delimitam área de abrangência com adstrição da clientela - e Unidades Básicas de Saúde (UBS) - em que a demanda atendida é espontânea ou encaminhada por outros serviços - sugere que a origem dessas diferenças pode se relacionar ao conceito de APS* proposto por Starfield ${ }^{33,34}$.

\footnotetext{
*Dimensões de avaliação para a atenção primária à saúde: acessibilidade, porta de entrada; elenco de serviços; vínculo; coordenação e orientaçâo familiar, orientação comunitária e formaçáo profissional.
} 
A necessidade de matriciamento em saúde mental, sua viabilidade política-institucional e o território onde ela acontece formam o trio de vetores apontados pelo levantamento realizado. Nesse sentido, duas experiências que ocorreram em períodos que antecedem a formalização dos CAPS, ESF e NASF se destacam: a de Sobral e a de Campinas. A primeira acontece através da preocupação com direitos humanos a partir de uma situação explícita de sua violação e a segunda surge de movimento intelectual local.

\section{A experiência de Sobral, CE}

Em 1999, na cidade de Sobral, em decorrência de violação de direitos humanos, morre paciente internado em hospital psiquiátrico. A denúncia resultou no fechamento do hospital e alavancou a prática de matriciamento ${ }^{35,9}$. Na época, acontecia nessa cidade o movimento de integração da saúde mental e Programa Saúde da Família, o qual buscava cuidar dos casos do território através de interconsultas e consultas conjuntas. O papel do CAPS da cidade foi fundamental, pois se tratava da retaguarda especializada dessa ação. Essa é uma das 12 experiências presentes no manual da OMS sobre integração entre Saúde Mental e a APS.

\section{A experiência de Campinas, SP}

Essa experiência se inicia em 2001, através do Programa Paidéia Saúde da Família, uma adaptação do Programa Saúde da Família do Ministério da Saúde, ajustado ao contexto sanitário local. A experiência de Campinas nasce em meio à discussão da saúde coletiva e do movimento intelectual na área dos trabalhadores de saúde mental que, desde 1997, através de publicação sobre metodologia de gestão de cuidado ${ }^{16}$, introduz o conceito de matriciamento e apoio matricial. A problemática tem origem na saúde coletiva e constrói referências do modelo de saúde pública brasileiro. Essa experiência do Programa Saúde da Família, pelos princípios de atenção integral ao sujeito e o vínculo, incorpora a dimensão subjetiva e social na prática clínica ${ }^{36}$.

\section{Conclusão}

Através da análise dos mecanismos de articulação entre Saúde Mental e Atenção Primária à Saúde no Brasil, publicados entre 2000 e 2010, observou-se que:

- Há concentração de publicaçóes nas regiôes Sudeste, Sul e em poucos estados do Nordeste, o que aponta o viés da iniquidade regional das publicaçóes e da oferta de serviços;

- A Saúde Mental e a Atenção Primária, embora sejam ambas de base territorial, têm diferenças metodológicas e isso tem consequência nas leituras, demandas e avaliaçóes sobre o trabalho em colaboraçáo e a prática do matriciamento;

- A operacionalização da Lei n. 10.216/2001 e das portarias n. 648/2006 e n. 154/2008 de forma articulada é um exercício que depende também do território onde ela ocorre;

- A prática do matriciamento é um convite para repensar o papel da reforma sanitária, representada pela ESF, e da reforma psiquiátrica, representada pelo CAPS, assim como o papel das Unidades de Saúde da Família, ambulatórios de saúde mental e hospitais.

Concluindo, embora a experiência brasileira esteja se construindo em consonância com as recomendaçóes mundiais que propóem diminuir a lacuna terapêutica entre oferta e necessidades de cuidado, a construção da Rede de Saúde Mental integrada à Estratégia Saúde da Família é um desafio novo para a reforma psiquiátrica brasileira. Daí a importância da análise: a estratégia de matriciamento corresponderá às expectativas de cobertura e acesso? 


\section{Referências}

1. Kohn R, Saxena S, Levav I, Saraceno B. The treatment gap in mental health care. Bull World Health Organ. 2004 Nov; 82(11): 858-866.

2. Whiteford H, Knaap M, Thornicroft G, Saxena S. Resources for mental health: scarcity, inequity, and inefficience. Lancet. 2007 Sep; 370 (9590): 878-89. http://dx.doi.org/10.1016/S0140-6736(07)61239-2

3. Weiss MG. Reducing the stigma of mental illness: a report from a global programme of the World Psychiatric Association. Bull World Health Organ. 2006 Apr; 84(4): 333-334.

4. Global Mental Health: The Lancet Series. London; 2007.

5. Thornicroft G, Tansella M. Quais são os argumentos a favor da atenção comunitária à saúde mental?. Pesqui Prát Psicossociais. $2008 ; 3(1): 9-25$.

6. Konh R, Saraceno B, Levav I, Saxena S. The treatment gap in mental health care. Bull World Health Organ. 2004; 82(11): 858-866. http://dx.doi. org/10.1590/S0042-96862004001100011

7. Prince M, Patel V, Saxena S, Maj M, Maselko J, Phillips MR et al. No health without mental health. Lancet. 2007 Sep 8; 370(9590): 859-77. http:// dx.doi.org/10.1016/S0140-6736(07)61238-0

8. Lancet Global Mental Health Group, Chisholm D, Flisher AJ, Lund C, Patel V, Saxena S, et al. Scale up services for mental disorders: a call for action. Lancet. 2007 Oct; 370(9594): 1241-52. http://dx.doi.org/10.1016/S0140-6736(07)61242-2

9. World Health Organization - WHO, World Organization of Family Doctors - WONCA. Integração da saúde mental nos cuidados de saúde primários: Uma perspectiva global. Lisboa: WHO, WONCA; 2009.

10. Bartels, Coakley EH, Zubritsky C, Ware JH, Miles KM, Areán PA, et al. Improving Access to Geriatric Mental Health Services: A Randomized Trial Comparing Treatment Engagement With Integrated Versus Enhanced Referral Care for Depression, Anxiety, and At-Risk Alcohol Use. Am J Psychiatry. 2004; 161:1455-1462. http://dx.doi.org/10.1176/appi.ajp.161.8.1455

11. Gask L, Bower P, Lovell K, Escott D, Archer J, Gilbody S, et al. What work has to be done to implement collaborative care for depression? Process evaluation of a trial utilizing the Normalization Process Model. Implement Sci. 2010 Feb; 5: 15. http://dx.doi.org/10.1186/1748-5908-5-15

12. A Report of The Collaborative Working Group on Shared Mental Health Care. Canadian Psychiatric Association, College of Family Physicians of Canada; 2000 .

13. Arona E. Implantação do matriciamento nos serviços de saúde de Capivari. Saúde Soc. 2009; 18(Suppl. 1): 26-36. http://dx.doi.org/10.1590/ S0104-12902009000500005

14. Barban E, Oliveira A. O modelo de assistência da equipe matricial de saúde mental no Programa Saúde da Família do município de São José do Rio Preto. Arq Ciênc Saúde; 2007 jan./mar.;14(1):54-65.

15. Canadian Collaborative Mental Health Conference. The College of Family Physicians of Canada. [acesso em 2011 nov.]. Disponível em http://www.sharedcare.ca/page. asp $\times$ ?menu $=57 \&$ app $=251 \&$ cat $1=628 \& t p=2 \& 1 \mathrm{k}=$ no

16. Campos GWS. Apoio matricial e equipe de referência: uma metodologia para a gestão do trabalho interdisciplinar em saúde. Cad Saúde Pública. 2007 fev.; 23(2): 399-407. http://dx.doi.org/10.1590/S0102-311X2007000200016

17. Brasil. Ministério da Saúde. Secretária de Atenção à Saúde. Portaria nº 154, de 24 de janeiro de 2008. Cria os Núcleos de Apoio à Saúde da Família - NASF. Diário Oficial da República Federativa do Brasil, Brasília,jan. 2008.

18. Brasil. Governo Federal. Lei no 10.216, de 06 de abril de 2001. Dispõe sobre a proteção e os direitos das pessoas portadoras de transtornos mentais e redireciona o modelo assistencial em saúde mental. Diário Oficial da República Federativa do Brasil, Brasília, abr. 2001.

19. Weber R, Vieira SF, Godinho Delgado PG. Política de Saúde Mental do Brasil - evolução do gasto federal entre 2001 e 2009 . Rev Saúde Pública. 2012; 46(1): 51-58. http://dx.doi.org/10.1590/S0034-89102011005000085

20. Greenhalgh T. Meta-narrative mapping: a new approach to the systematic review of complex evidence. In: Hurwitz B, Greenhalgh T, Skultans V. Narrative Research in Health and Illness. BMJ Books; 2004.

21. Noyes J, Popay J, Pearson A, Hannes K, Booth A. Qualitative research and Cochrane reviews. In: Higgins P, Green S. Cochrane Handbook for Systematic Reviews of Interventions: Cochrane Book Series. Chichester: John Wiley \& Sons, Ltd; 2008. cap. 20. http://dx.doi.org/10.1002/9780470712184.ch20

22. Nascimento A, Braga V. Atenção em Saúde Mental: a prática do enfermeiro e do médico do Programa Saúde da Família de Caucaia - CE. Cogitare Enferm. 2004 jan./jun.; 9(1): 84-93.

23. Carneiro A, Oliveira ACM, Santos MMS, Alves MS, Casais NA, Santos JE. Saúde mental e atenção primária: uma experiência com agentes comunitários de saúde em Salvador-BA. Rev Bras Promoç Saúde. 2009 out./dez.; 22(4): 264-271. http://dx.doi.org/10.5020/18061230.2009.p264

24. Coyne JC, Schwenk TL, Fechner-Bates S. Non detection of Depression by Primary Care Physicians Reconsidered. Gen Hosp Psychiatry. $1995 ; 17(1): 3-12$. http://dx.doi.org/10.1016/0163-8343(94)00056-J

25. Ustun TB, Sartorius N. Mental IIness in General Health Care: na International Study. Chichesser: John Wiley \& sons; 1995.

26. Mari J. Minor Psychiatric Morbidity in Three Primary care Clinics in the City of São Paulo. Soc Psychiatry Psychiatr Epidemiol. 1987; 22:129-138. http://dx.doi.org/10.1007/BF00583847

27. Mari J, Willians P. A Comparison of the Validity of Two Psychiatric Screening Questionnaires (GHQ-12 and SRQ-20) in Brazil Using Relative Operating Characteristic (ROC) Analysis. Psychol Med. 1985 Aug; 15(3): 651-9. http://dx.doi.org/10.1017/S0033291700031500 
28. Vilano L. Problemas Psicológicos e morbidades psiquiátricas em serviços de saúde não psiquiátricos: o ambulatório de clinica geral. [Tese]. São Paulo: Escola Paulista de Medicina, Universidade Federal de São Paulo; 1998. 227 p.

29. Lacoponi E. The Detection of Emotional Disorders by Primary Care Physicians - a Study in São Paulo, Brazil. [Thesis]. London: University of London; 1989

30. Mari J, lacoponi E, Williams P, Simões O, Silva JB. Detection of psychiatric morbidity in the primary medical care setting in Brazil. Rev Saúde Públ. 1987; 21:501-7. http://dx.doi.org/10.1590/S0034-89101987000600006

31. Fortes S, Villano LAB, Lopes CS. Nosological profile and prevalence of common mental disorders of patients seen at the Family Health Program (FHP) units in Petrópolis, Rio de Janeiro. Rev Bras Psiquiatr. 2008 Mar; 30(1): 32-37. http://dx.doi.org/10.1590/S1516-44462006005000066

32. Elias E, Ferreira CW, Alves MCG, Cohn A, Kishima V, Escrivão Júnior A, et al. A atenção Básica em saúde: comparação entre PSF e UBS por extrato de exclusão social no município de São Paulo. Ciênc Saúde Coletiva. 2006; 11(3): 633-641. http://dx.doi.org/10.1590/S1413-81232006000300012

33. Starfield B. Atenção primária: equilíbrio entre necessidades de saúde, serviços e tecnologia. Brasília: UNESCO Brasil, Ministério da Saúde; 2004.

34. Macincko J, Almeida C, Oliveira E, Sá P. Organization and delivery of primary health care services in Petrópolis, Brazil. Int J Health Plann Mgmt. 2004; 19: 303-17.

35. Tofoli LF, Fortes S. Apoio Matricial de Saúde Mental na Atenção Primária no Município de Sobral no Ceará: relato de experiência. Sanare. $2007 ; 6(2): 34-42$.

36. Figueiredo Dorsa M, Onocko R. Saúde Mental e Atenção Básica à Saúde: o apoio matricial na construção de uma rede multicêntrica. Saúde Debate. 2008; 32(78-80): 143-150 